\title{
Estado Nutricional e Prevalência de Síndrome Metabólica em Praticantes Amadores de Futebol
}

\author{
Nutritional Status and Prevalence of Metabolic Syndrome \\ in Amateur Soccer Players
}

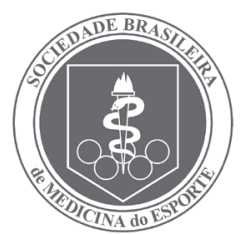

Artigo Original
Luciano Meireles de Pontes ${ }^{1,2,3}$ Maria do Socorro Cirilo de Sousa ${ }^{4}$

1. Mestre em Ciências da Nutrição - Programa de Pós-graduação Stricto Sensu em Ciências da Nutrição. Centro de Ciências da Saúde, Universidade Federal da Paraíba (UFPB).

2. Bolsista da Fundação de Amparo à Ciência e Tecnologia do Estado de Pernambuco (FACEPE) - Nível doutorado - Programa de Pósgraduação em Saúde da Criança e do Adolescente - Universidade Federal de Pernambuco (UFPE). 3. Laboratório de Avaliação Física (LAF) UNIPÊ/SANNY - Centro Universitário de João Pessoa (UNIPÊ). João Pessoa, PB. 4. Professora adjunta do Departamento de Educação Física. Centro de Ciências da Saúde - Departamento de Cultura Física e Reabilitação, Universidade Federal da Paraíba (UFPB). João Pessoa, PB.

Pesquisa aprovada sob protocolo no. 051/04 pela Comissão de Bioética do Centro de Ciências da Saúde (CCS) da Universidade Federal da Paraíba (UFPB).

Endereço para correspondência: Luciano Meireles de Pontes Rua Juvenal Mário da Silva, 894, Manaíra

58038-511 - João Pessoa, PB.

Tel.: (83) 3246-1448.

E-mail:

mslucianomeireles@gmail.com

Submetido em 04/08/2008 Versão final recebida em 29/10/2008 Aceito em 28/11/2008

\section{RESUMO}

O objetivo do estudo foi avaliar o estado nutricional e identificar a prevalência de síndrome metabólica em praticantes amadores de futebol. Foram estudados 32 homens (39,0 \pm 6,4anos) com prática habitual de futebol. O estado nutricional foi avaliado por meio do índice de massa corporal (IMC) e a classificação da World Health Organization; a síndrome metabólica, pelo critério da International Diabetes Federation, que considera para o sexo masculino, além da obesidade central (circunferência da cintura $>90 \mathrm{~cm}$ ), duas ou mais das seguintes condições: trigliceridemia $\geq 150 \mathrm{mg} / \mathrm{dl}$; níveis de $\mathrm{HDL}<40 \mathrm{mg} / \mathrm{dl}$; pressão sistólica $\geq 130 \mathrm{mmHg}$ ou diastólica $\geq 85 \mathrm{~mm} / \mathrm{Hg}$; e glicemia em jejum $\geq 100 \mathrm{mg} / \mathrm{dl}$. A análise estatística processou dados descritivos de percentuais, média, mínimo, máximo, desvio padrão e inferência de correlações entre pares de variáveis por meio do coeficiente $r$ de Pearson. O nível de significância adotado foi de $p<0,05$. Os resultados encontrados mostraram que $43,8 \%$ dos futebolistas são eutróficos, $43,8 \%$ têm sobrepeso e 12,5\%, obesidade. A ocorrência de síndrome metabólica foi de $37,5 \%$ e seus componentes mais prevalentes foram: 59,4\% obesidade abdominal, 40,6\% hipertensão, 34,4\% hipertrigliceridemia, 28,1\% HDL-C e 15,6\% glicemia em jejum. O IMC apresentou correlação mais robusta com a circunferência da cintura $(r=0,918)$. Conclusão: Os futebolistas investigados apresentaram ocorrência de sobrepeso e obesidade em seu estado nutricional e, apesar de a frequência de síndrome metabólica se apresentar inferior a de outros estudos epidemiológicos, acredita-se que, além da prática de atividade física, esses desportistas devem aderir a outros comportamentos promotores de saúde, como a adesão a bons hábitos alimentares e ao controle do estresse.

Palavras-chave: futebol, sobrepeso, obesidade, síndrome metabólica.

\section{ABSTRACT}

The objective of the study was to evaluate the nutritional status and to identify the prevalence of metabolic syndrome in amateur soccer practitioners. 32 men (39.0 \pm 6.4 years) were studied with habitual soccer practice. Nutritional status was evaluated through Body Mass Index (BMI) and the World Health Organization guidelines, and the metabolic syndrome by the International Diabetes Federation criterion that considers for the male sex, besides central obesity (waist circumference $>90 \mathrm{~cm}$ ), two or more of the following conditions: triglyceridemia $\geq 150 \mathrm{mg} / \mathrm{dl}$; HDL levels $<40 \mathrm{mg} / \mathrm{dl}$; systolic pressure $\geq 130 \mathrm{mmHg}$ or diastolic $\geq 85 \mathrm{~mm} / \mathrm{Hg}$; and fasting glycemia $\geq 100 \mathrm{mg} / \mathrm{dl}$. Statistical analysis processed descriptive data of percentages, mean, minimum, maximum, standard deviation and inference of correlations between variable pairs through the "r" coefficient of Pearson. Significance level adopted was of $p<0.05$. The found results showed that $43.8 \%$ of the soccer players are eutrophic, $43.8 \%$ are overweighed and $12.5 \%$ are obese. Occurrence of metabolic syndrome was $37.5 \%$ and their most prevalent components were: $59.4 \%$ abdominal obesity, 40.6\% hypertension, 34.4\% hypertriglyceridemia, 28.1\% HDL-C and 15.6\% fasting glycemia. BMI presented more remarkable correlation with waist circumference $(r=0.918)$. Conclusion: The investigated soccer players presented overweight and obesity occurrence in their nutritional status and in spite of lower frequency of metabolic syndrome here than in other epidemic studies, it is believed that besides the practice of physical activity, these sportsmen should adopt other healthy behavior such as good eating habits and stress control.

Keywords: soccer, overweight, obesity, metabolic syndrome. 


\section{INTRODUÇÃO}

A síndrome metabólica representa uma condição clínico-epidemiológica bastante estudada nos últimos anos. Apesar de alguns desencontros em relação a sua definição, pois não há uma descrição internacional definitiva, conceitualmente, caracteriza-se pela associação, num mesmo indivíduo, de diabetes mellitus tipo 2, ou intolerância à glicose, hipertensão arterial, dislipidemia e obesidade abdominal ${ }^{(1,2)}$.

A partir de 1999 foi sugerido, pela World Health Organization $(\mathrm{WHO})^{(3)}$, uma definição fundamentada em dados clínicos e laboratoriais que pudessem ser medidos com relativa facilidade (glicemia de jejum, resistência à ação da insulina, pressão arterial, circunferência da cintura e do quadril, índice de massa corporal, triglicerídeos, HDL-C, microalbuminúria). Em 2001, o National Cholesterol Education Program - Adult Treatment Panel III (NCEP-ATP III)(4) propôs uma série de critérios semelhantes, porém mais simples de serem avaliados, incluindo: glicemia de jejum, pressão arterial, cintura, triglicerídeos e HDL-C. Outras definições surgiram com a American Heart Association $^{(5)}$ e o European Group for the Study of Insulin Resistance ${ }^{(6)}$. Recentemente, a International Diabetes Federation (IDF) ${ }^{(7)}$ vem discutindo uma nova definição, na qual a obesidade abdominal é um dos elementos centrais e sugere pontos de corte específicos para diferentes grupos étnicos. Notadamente, a prevalência da síndrome metabólica nas diferentes populações é fortemente dependente dos critérios utilizados para sua definição. Dessa forma, alguns pesquisadores, entre eles Ford e Giles ${ }^{(8)}$ e Jorgensen et al. ${ }^{(9)}$ sugerem que uma classificação universal seja adotada para facilitar comparações entre estudos. Na presente pesquisa, optou-se pela definição adotada pelo recente padrão da IDF ${ }^{(7)}$, que representa uma fusão dos três últimos referenciais produzidos pela $\mathrm{WHO}^{(3)}$, NCEP-ATP III (4) e do grupo de estudo sobre resistência insulínica ${ }^{(6)}$. Tal proposta é mais próxima da do NCEP-ATP $I^{\left({ }^{(4)}\right.}$, que inclui as mesmas variáveis, diferindo ao considerar a obesidade central como um componente essencial; além disso, a circunferência da cintura é fixada a um nível mais baixo do apresentado pelo NCEP-ATP III (4).

Com o intuito de minimizar e prevenir os efeitos do excesso de peso, órgãos e sociedades de saúde ${ }^{(10-12)}$ vêm indicando a atividade física como importante componente de prevenção de doenças cardiovasculares. Assim, é consenso que, independente da supervisão e controle periodizado, a atividade física é salutar e pode ser um componente protetor para várias doenças crônicas não transmissíveis ${ }^{(13)}$. Entretanto, nota-se que mesmo populações com histórico habitual de prática esportiva podem estar expostas aos padrões de desequilíbrio nutricional, levando ao excesso de peso; essa condição pode estar ligada ao descuido de outros comportamentos do estilo de vida.

Sobre a prática desportiva, é cada vez maior o contingente de indivíduos que buscam se exercitar nos momentos de lazer, sobretudo praticando esportes com caráter recreativo e sem fins competitivos. No entanto, nota-se baixa adesão desses desportistas na prática do esporte ou baixa frequência, já que muitas vezes as atividades são realizadas apenas nos finais de semana. Esse perfil é visto em diversas modalidades esportivas, como é o caso do futebol. Esses desportistas ficam expostos ao desenvolvimento de distúrbios nutricionais, possivelmente por ser insuficientemente ativos. Ao observar a literatura, nota-se que ainda é pertinente buscar informações sobre a obesidade, suas características e efeitos, principalmente em praticantes de algum tipo de prática esportiva amadora, já que o perfil desses desportistas ainda não é totalmente conhecido. Assim, justifica-se a realização deste estudo, que objetiva avaliar o estado nutricional e identificar a prevalência de síndrome metabólica em praticantes amadores de futebol.

\section{MÉTODOS}

O delineamento deste estudo foi transversal por observar fator e efeito num mesmo momento histórico e utilizou abordagem quantitativa. A população estudada foi constituída por futebolistas frequentadores da área de esportes do Centro Federal de Educação Tecnológica da Paraíba (Cefet) localizado em João Pessoa. Foram investigados 32 homens (39,0 $\pm 6,4$ anos). Os desportistas foram selecionados pelo método probabilístico aleatório simples por meio de sorteio a partir da listagem de 60 inscritos no departamento de Educação Física da instituição. Os critérios de inclusão foram: ter prática habitual de futebol com caráter amador nos últimos seis meses (informação referida), aceitar as normas e participação voluntária, submetendo-se às avaliações propostas na pesquisa. Para melhor caracterizar os participantes da pesquisa, optou-se por coletar também informações referentes ao padrão econômico por meio de questionário desenvolvido pela ABA/ Abipeme e adaptado por Almeida e Wickerhauser ${ }^{(14)}$, com escalas de classificação subdivididas em cinco categorias: A/B (alta), C (média), D (baixa), E (muito baixa), de acordo com o grau de instrução do chefe da família e os bens de consumo familiar, e da predominância da cor da pele.

\section{Variáveis do estado nutricional e síndrome metabólica}

Antropometria: A massa corporal foi mensurada com o indivíduo com o mínimo de roupa (short/sunga), posicionado na plataforma de uma balança digital (Camry, São Paulo) com capacidade entre zero e 150kg e divisão aproximada a 100g; a medida de estatura foi obtida por meio de um estadiômetro portátil (American Medical do Brasil, São Paulo) com tolerância de $\pm 2 \mathrm{~mm}$ a $204 \mathrm{~cm}$, sendo considerada a distância do vértex à região plantar, com o indivíduo posicionado em pé sobre a base do equipamento, cabeça orientada no plano frontal, membros superiores ao longo do corpo, pés unidos, as superfícies posteriores dos calcanhares, cintura pélvica, cintura escapular e região occipital em contato com a escala de medida. 0 estado nutricional foi definido pelo índice de massa corporal (IMC) e padrão da WHO ${ }^{(15)}$, considerando: IMC entre 18,5 e 24,9kg/m², "eutrofia"; IMC entre 24,9 kg/m² e 29,9 kg/m², "sobrepeso"; e IMC superior a $30,0 \mathrm{~kg} / \mathrm{m}^{2}$, "obesidade".

Obesidade abdominal: foi analisada a circunferência medida no ponto abdominal (CA) por meio de trena modelo Sanny (American Medical do Brasil, São Paulo); as mensurações foram realizadas com o sujeito de frente para o avaliador, a medida foi determinada no mesmo plano horizontal à altura da cicatriz umbilical. Valores superiores a $90 \mathrm{~cm}$ foram considerados de risco, conforme as diretrizes da $\operatorname{IDF}^{(7)}$ e o padrão para a América do Sul.

Pressão arterial: Foi aferida através de um esfignomanômetro (BD, Juiz de Fora) e um estetoscópio tipo Rappaport (Premium, Curitiba), testados e calibrados anteriormente. A classificação dos níveis tensionais seguiu o padrão mencionado nas $V$ Diretrizes Brasileiras de Hipertensão Arterial(16) , com a seguinte descrição: o indivíduo na posição sentada após 10 minutos de repouso, braço direito apoiado e ao nível do coração, colocou-se a braçadeira do aparelho cerca de $3 \mathrm{~cm}$ acima da fossa antecubital, centralizando a bolsa de borracha sobre a artéria umeral; procedeu-se à desinsuflação da braçadeira com velocidade constante. Visando minimizar a "síndrome do jaleco branco", além do repouso antes da aferição, a medição foi realizada duas vezes com o intervalo de cinco minutos, sendo considerada a média das duas medições. As medidas pressóricas foram realizadas por avaliador experiente e conhecedor de todos os procedimentos de avaliação. 
Dosagens sanguíneas: As dosagens foram realizadas através da coleta de sangue venoso na prega do cotovelo, após um período de 8-10h em jejum, entre 7h30 e 8h30. O soro foi separado por centrifugação, sendo determinados os teores de triglicérides através do método enzimático com lipase, colesterol de alta densidade (HDL-C) pelo método enzimático-colorimétrico do colesterol esterase/colesterol oxidase e glicose em jejum através do método da glicose-oxidase processados em analisador enzimático por kits da marca Doles Glucox 500. As dosagens foram realizadas em um único laboratório indicado pelos pesquisadores, devidamente equipado, credenciado e localizado em João Pessoa.

A síndrome metabólica foi considerada presente a partir dos critérios descritos pela IDF expostos no quadro 1:

Quadro 1. Critérios IDF para identificação da síndrome metabólica

\begin{tabular}{|l|l|}
\hline 1.Obesidade central & $C A \geq 90 \mathrm{~cm}$ \\
\hline $\begin{array}{l}\text { 2. Duas ou mais das seguintes } \\
\text { condições: }\end{array}$ & a) trigliceridemia $\geq 150 \mathrm{mg} / \mathrm{dl}$ \\
\cline { 2 - 2 } & b) níveis de $\mathrm{HDL}<40 \mathrm{mg} / \mathrm{dl}$ (homens) \\
\hline & $\begin{array}{l}\text { c) pressão sistólica } \geq 130 \mathrm{mmHg} \text { ou diastólica } \geq \\
85 \mathrm{~mm} / \mathrm{Hg}\end{array}$ \\
\hline & d) Glicemia de jejum $\geq 100 \mathrm{mg} / \mathrm{dl}$ \\
\hline
\end{tabular}

\section{COLETA DE DADOS}

Inicialmente foi realizado o contato, seguido de autorização da direção do Cefet para a realização do estudo. Os sujeitos, também contatados e selecionados randomicamente, foram informados dos procedimentos, dos possíveis desconfortos, riscos e benefícios do estudo, antes de assinar um termo de consentimento livre e esclarecido, segundo as normas para realização de Pesquisa em Seres Humanos e os critérios de Ética em Pesquisa - Resolução 196/96. Em seguida, foram realizadas as mensurações de pressão arterial, antropometria e respondidos os questionários de classificação econômica, etnia e coleta em laboratório. A equipe básica para coleta de dados foi composta por um profissional de Educação Física com especialidade em avaliação morfofuncional para a mensuração das medidas antropométricas e pressão arterial; e uma enfermeira para a coleta sanguínea, capacitada por uma clínica de referência da cidade de João Pessoa. O protocolo de estudo foi aprovado sob nº. 051/04 pela Comissão de Ética em Pesquisa do Centro de Ciências da Saúde da Universidade Federal da Paraíba. A análise estatística processou dados descritivos de percentuais, média, mínimo, máximo, desvio padrão (DP) e inferência de correlações entre pares de variáveis por meio do coeficiente $r^{\prime \prime}$ de Pearson. Para tais procedimentos foi utilizado o programa SPSS em sua versão 16.0.

\section{RESULTADOS}

Os participantes deste estudo praticavam futebol com caráter amador/recreativo habitualmente nos últimos seis meses com uma frequência de pelo menos uma vez por semana. Para melhor identificar a amostra identificou-se o nível sócio-econômico dos futebolistas e a predominância da cor da pele. Observou-se que: $81,3 \%$ são da classe B e 18,7\% C; Em relação à cor da pele, 71,9\% são caucasianos, 25,0\% mestiços e 3,1\% negros.

Na tabela 1, observam-se as variáveis antropométricas, fisiológicas e metabólicas. Foram identificadas médias de IMC $\left(25,9 \mathrm{~kg} / \mathrm{m}^{2}\right)$ e CA $(91,5 \mathrm{~cm})$ acima dos valores recomendados para boa situação nutricional, ou seja, indicando sobrepeso e obesidade abdominal.
Tabela 1. Distribuição dos valores de média, DP, mínimo e máximo das variáveis antropométricas, fisiolólogicas e metabólicas dos praticantes amadores de futebol $(n=32)$.

\begin{tabular}{l|c|c|c}
\hline Variáveis antropométricas & Média \pm DP & Mínimo & Máximo \\
\hline Massa corporal (kg) & $76,5 \pm 11,9$ & 48,8 & 106,3 \\
\hline Estatura (m) & $1,71 \pm 0,7$ & 1,52 & 1,83 \\
\hline IMC (kg/m²) & $25,9 \pm 3,2$ & 18,8 & 31,6 \\
\hline CA (cm) & $91,5 \pm 9,5$ & 66,5 & 113,5 \\
\hline Fisiológicas/metabólicas & & & \\
\hline PAS (mg/Hg) & $126,9 \pm 12,1$ & 102 & 160 \\
\hline PAD (mg/Hg) & $82,7 \pm 7,6$ & 60 & 100 \\
\hline Glicemia em jejum (mg/dl) & $89,7 \pm 9,1$ & 72,0 & 112,0 \\
\hline HDL-C (mg/dl) & $49,8 \pm 10,9$ & 29 & 78 \\
\hline Triglicérides (mg/dl) & $141,0 \pm 74,4$ & 50,0 & 315,0 \\
\hline
\end{tabular}

A tabela 2 expõe os dados do estado nutricional. Observou-se percentual elevado de excesso de peso, já que $43,7 \%$ se enquadraram como sobrepeso e 12,6\% como obesidade.

Tabela 2. Distribuição percentual do estado nutricional dos praticantes amadores de futebol $(n=32)$.

\begin{tabular}{l|c|c}
\hline \multicolumn{1}{c|}{ Estado nutricional } & $\mathbf{n}$ & $\%$ \\
\hline Eutrófico & 14 & 43,7 \\
\hline Sobrepeso & 14 & 43,7 \\
\hline Obesidade & 04 & 12,6 \\
\hline Total & 32 & 100,0 \\
\hline
\end{tabular}

A tabela 3 apresenta a distribuição das prevalências da síndrome metabólica e seus componentes de risco. Os fatores mais observados foram: 59,4\% para obesidade abdominal, 40,6\% para hipertensão e $34,4 \%$ para hipertrigliceridemia. O HDL-C e a glicemia de jejum também apresentaram valores percentuais de alerta. Foi observado que 37,5\% dos indivíduos apresentam três ou mais fatores de risco para a síndrome metabólica.

Tabela 3. Análise da prevalência e componentes de risco para síndrome metabólica em praticantes de futebol $(n=32)$.

\begin{tabular}{l|c|c}
\hline Síndrome metabólica e componentes de risco & $\mathbf{n}$ & $\%$ \\
\hline Presença de síndrome metabólica (Critério IDF $\left.{ }^{7}\right)$ & 12 & 37,5 \\
\hline Normalidade & 20 & 62,5 \\
\hline Obesidade abdominal (CA $\geq 90 \mathrm{~cm})$ & 19 & 59,4 \\
\hline Trigliceridemia $\geq 150 \mathrm{mgdl}$ & 11 & 34,4 \\
\hline HDL $<40 \mathrm{mmdl}($ sexo masculino) & 9 & 28,1 \\
\hline PAS $\geq 130 \mathrm{mmHg}$ ou PAD $\geq 85 \mathrm{mmHg}$ & 13 & 40,6 \\
\hline Glicemia em jejum $\geq 100 \mathrm{mg} / \mathrm{dl}$ & 5 & 15,6 \\
\hline Presença de fatores de risco (FR) & $n$ & $\%$ \\
\hline Nenhum & 9 & 28,1 \\
\hline Um FR & 5 & 15,6 \\
\hline Dois FR & 6 & 18,8 \\
\hline Três FR & 8 & 25,0 \\
\hline Quatro ou mais FR & 4 & 12,5 \\
\hline
\end{tabular}


Entre as correlações vistas na tabela 4, a CA mostrou a relação mais robusta $(r=0,918)$ com a variável do estado nutricional (IMC). 0 HDL-C apresentou tendência inversa à do índice de Quetelet; os outros indicadores mostraram valores positivos, mais baixos estatisticamente $(r<0,60)$.

Tabela 4. Correlação entre o IMC e os componentes da síndrome metabólica nos praticantes amadores de futebol $(n=32)$.

\begin{tabular}{l|c|c}
\hline Componentes da sindrome metabólica & \multicolumn{2}{|c}{ IMC } \\
\hline & "r" & $p$ \\
\hline CA & $0,918^{*}$ & $0,000^{* *}$ \\
\hline Trigliceridemia & 0,408 & $0,020^{* *}$ \\
\hline HDL-C & $-0,444$ & $0,011^{* *}$ \\
\hline PAS & 0,390 & $0,010^{* *}$ \\
\hline PAD & 0,570 & $0,001^{* *}$ \\
\hline Glicemia em jejum & 0,259 & 0,152 \\
\hline
\end{tabular}

* Correlação linear de Pearson (elevada) * ${ }^{* *}<0,05$ (significativo).

\section{DISCUSSÃO}

A prevalência da síndrome metabólica tem sido descrita em diferentes grupos étnicos e populações de vários países. No entanto, existem poucos estudos com amostras em nível populacional realizados em diferentes etnias que possam representar a realidade de cada povo. Algumas pesquisas nacionais e internacionais, como no semiárido baiano (Brasil)(17), no México ${ }^{(18)}$, nos Estados Unidos ${ }^{(19)}$ e no Japão(20) , têm revelado prevalências variadas. Dependendo do critério utilizado e das características da população investigada, essas taxas variam entre $12,4 \%$ e 41,2\% para o sexo masculino.

Não está ainda determinado qual é a melhor definição operacional da síndrome metabólica para ser utilizada na prática clínica; segundo os especialistas, o ideal seria levar em conta a aplicabilidade clínica e o desenvolvimento de desfechos. No presente estudo, identificou-se a síndrome metabólica pela classificação europeia do IDF(7) por considerar em seus critérios diferenças étnicas de acordo com o continente de cada população (América, África, Ásia, Europa e Oceania).

De acordo com o I Consenso Brasileiro de Síndrome Metabólica(21), ainda não se têm estudos de prevalência com dados representativos da população brasileira, embora algumas pesquisas regionalizadas venham sendo publicadas.

Sobre a prevalência de síndrome metabólica aqui encontrada, foi considerada elevada, quando comparada com os resultados de Oliveira et al. ${ }^{(17)}$, que encontraram 18,6\% em homens do semiárido baiano. Em parte, essa alta taxa pode ter ocorrido pelo critério de diagnóstico que foi adotado, já que os pontos de corte da IDF(7) contemplam valores menores no perímetro da cintura em relação aos níveis propostos pela $\mathrm{WHO}^{(3)}$ e NCEP-ATP III(4), identificando, assim, maior número de acometidos.

Em pesquisa realizada por Vargas et al. ${ }^{(18)}$, em Oaxaca, no México, foram investigados 325 homens maiores de 35 anos aparentemente saudáveis, com o objetivo de esclarecer as associações da síndrome metabólica e estilo de vida. Os autores utilizaram os mesmos critérios adotados nesta pesquisa, os da IDF, e os resultados apontaram prevalência de 41,2\% de síndrome metabólica nos indivíduos da zona urbana, percentuais mais altos do que os deste estudo.

Gupta et al.(22), em estudo de prevalência realizado na Índia, encontraram 21,0\% de síndrome metabólica em 532 homens com baixos níveis de adesão à prática de exercícios físicos. Os participantes da pesquisa apresentavam características de atividade física similares às de futebolistas aqui estudados. Resultado semelhante foi, também, encontrado por Ninomiya et al. ${ }^{(20)}$ que, objetivando associar a síndrome metabólica ao risco cardiovascular, utilizando os critérios do NCEPATP III (4), encontraram valores prevalentes de 21,0\% em asiáticos de meia-idade. Em relação aos componentes da síndrome, destacou-se a obesidade abdominal, que é caracterizada pelo acúmulo de gordura visceral na região do tronco. Novamente, deve-se ressaltar que a alta ocorrência de gordura centralizada vista nos futebolistas, provavelmente, se deva à exigência da IDF, que estabelece pontos de corte mais rigorosos e diferenciados de acordo com a característica demográfica de cada população. Outros fatores de risco mais evidenciados foram a hipertensão arterial e a hipertrigliceridemia.

Medina-Lezama et al.(23), em estudo realizado no Peru com 1.878 indivíduos com baixa adesão a atividade física, utilizaram a classificação do American Heart Association e encontraram como componentes mais presentes os triglicerídeos elevados (52,0\%), baixo HDL-C (32,5\%) e a obesidade abdominal (14,0\%).

A glicose em jejum foi o indicador menos prevalente nos futebolistas, o que corroborou os achados da pesquisa realizada no Peru. No estudo de Gupta et al. ${ }^{(22)}$ os percentuais mais evidentes foram baixo HDL-C (46,6\%) e níveis pressóricos elevados (26,8\%).

Quando analisado o conjunto de fatores de risco, observou-se frequente acometimento de futebolistas com três ou mais fatores de exposição para síndrome metabólica. O fato mais preocupante é que a presença desses fatores os expõe à morbidade cardiovascular e, em casos mais extremos, à morte súbita na prática do futebol. Essas informações reforçam os resultados expostos em outros estudos ${ }^{(24,25)}$.

Para Ciolac e Guimarães ${ }^{(26)}$, como os portadores de síndrome metabólica apresentam um conjunto de fatores de risco para doença cardiovascular e resistência insulínica, eles obterão maiores benefícios com a prática regular de atividade física se esta for planejada de forma individualizada, focalizando a diminuição desses componentes de maneira progressiva. Outras autores ${ }^{(27)}$ referem que pesquisas epidemiológicas têm demonstrado relação direta entre a realização de atividade física e a melhora dos múltiplos fatores de risco como os encontrados na síndrome metabólica.

Em estudo experimental recente, Pontes et al.(28) acompanharam futebolistas amadores que praticavam o futebol apenas um dia por semana durante 16 semanas, e concluíram que a prática esportiva não foi suficiente para impactar melhoras nos níveis do estado nutricional e da aptidão física dos praticantes. O elevado percentual de sobrepeso/ obesidade pode ser explicado pela baixa adesão ao futebol (frequência $\leq$ duas vezes por semana) realizada pelos pesquisados.

Santos et al.(29), em um modelo exploratório visando observar a prevalência da síndrome metabólica e verificar sua associação com o excesso de peso e inatividade física, utilizaram uma amostra por conveniência de 47 homens (34,6 anos) funcionários de empresas e estabelecimentos de ensino e observaram que todos os portadores de síndrome metabólica apresentavam excesso de peso ou obesidade. Esse perfil corrobora o estudo de Meigs et al..(30), que a partir dos critérios do NCEP-ATP III (4), avaliaram o risco de diabetes e doenças cardiovasculares através da incidência de obesidade, concluindo que 63,0\% dos obesos apresentam síndrome metabólica. As explicações dadas pelos epidemiologistas para o crescimento epidêmico da obesidade apontam para a modernização das sociedades, que, entre outras coisas, proporciona maior oferta de alimentos e desequilíbrio na qualidade da dieta, aliada à melhoria dos instrumentos de trabalho, que gera baixo nível de atividade física ocupacional e de lazer.

Com o intuito de perceber o comportamento dos indicadores característicos da síndrome metabólica e o IMC entre os participantes deste estudo, observaram-se as correlações estabelecidas nas variá- 
veis constituintes da síndrome metabólica. Com exceção do HDL-C, que mostrou correlação negativa (inversa), fato que demonstra a ação protetora do colesterol de alta densidade, que pode contribuir para a redução do peso corporal, encontraram-se relações positivas (diretas) com maior valor estatístico visto na CA. Em pesquisa realizada por Geloneze et al.(31), que incluiu aproximadamente 2.000 indivíduos com predomínio de obesos e diabéticos, constatou-se associação elevada e positiva entre os componentes tradicionais da síndrome metabólica com a presença da adiposidade centralizada. Para estudiosos como Carr e Brunzell(32), o perímetro da cintura e/ou abdominal são as medidas mais recomendadas para diagnósticos de anormalidade na composição corporal em estudos populacionais. Visto que são os preditores antropométricos mais representativos da gordura intra-abdominal e apresentam aferição simplista e reprodutível.

Algumas limitações desta pesquisa são, reconhecidamente, a desconsideração da medida de atividade física dos futebolistas que poderia melhor esclarecer sobre a presença de excesso de peso (sobrepeso/ obesidade) e síndrome metabólica, além do tamanho da amostra, que provavelmente pode ter interferido na predição dos achados. No entanto, os dados evidenciados apresentam tendência que pode ser referenciada em outros estudos epidemiológicos.

\section{CONCLUSÃO}

Os futebolistas que participaram desta pesquisa apresentaram prevalência de sobrepeso e obesidade em seu estado nutricional e, apesar de a frequência de síndrome metabólica se apresentar inferior à de outros estudos epidemiológicos, acredita-se que, além da prática de atividade física, esses desportistas devem aderir a outros comportamentos promotores de saúde, como bons hábitos alimentares e o controle do estresse.

Dentre os principais componentes da síndrome metabólica foi destacada a obesidade abdominal, condição preocupante devido à associação desse padrão de obesidade com várias doenças crônicas não transmissíveis.

Vale ressaltar que a comparabilidade dos dados sobre síndrome metabólica deve ser vista com cautela, devido à falta de padronização universal nos critérios de diagnóstico para populações generalizadas.

Por fim, novas pesquisas são necessárias para melhor elucidar a presença dos distúrbios nutricionais e metabólicos em indivíduos com diferentes níveis de prática de atividade física como os desportistas aqui investigados, já que melhor conhecimento desses problemas contribuirá para intervenções mais precoces e eficientes na prevenção da obesidade e síndrome metabólica. As novas pesquisas devem também estudar, com maior atenção, a influência do sedentarismo e de outros determinantes comportamentais e hereditários relacionados com o processo saúde-doença.

Todos os autores declararam não haver qualquer potencial conflito de interesses referente a este artigo.

\section{REFERÊNCIAS BIBLIOGRÁFICAS}

1. Zimmet P, Boyko EJ, Collier GR, Courten M. Etiology of the metabolic syndrome: potential role of insulin resistance, leptin resistance, and other players. Ann NY Acad Sci 1999;892:25-44.

2. Wilson PW, D Agostino RB, Parise H, Sullivan L, Meigs JB. Metabolic syndrome as a precursor of cardiovascular disease and type 2 diabetes mellitus. Circulation 2005;112:3066-72.

3. World Health Organization (WHO). Definition, diagnosis and classification of diabetes mellitus and its complications: Report of WHO a Consultation. Part 1: diagnosis and classification of diabetes mellitus. Genebra, 1999

4. Third Report of The National Cholesterol Education Program (NCEP) expert panel on detection, evaluation, and treatment of high blood cholesterol in adults (Adult Treatment Panel III). Circulation 2002;106:3143-421.

5. Grundy SM, Cleeman Jl, Daniels SR, Donato KA, Eckel RH, Franklin BA, et al. Diagnosis and management of the metabolic syndrome: An American Heart Association/National Heart, Lung, and Blood Institutes scientific statement. Circulation 2005;18:1-18.

6. Einhorn D, Reaven GM, Cobin RH, Ford E, Ganda OP, Handelsman Y, et al. ACE Position Statement on the Insulin Resistance Syndrome. Endocr Pract 2003;9:240-52.

7. International Diabetes Federation. IDF Worlwide definition of the metabolic syndrome. International Diabetes Federation. [Federação Internacional de Diabetes on line]. Maio; 12. Disponível em: <http:// www.idf.org/webdata/docs/IDF_Meta_def_final.pdf> [2008 maio 11].

8. Ford ES, Giles WH. A comparison of the prevalence of the metabolic syndrome using two proposed definitions. Diabetes Care 2003;26:575-81

9. Jorgensen ME, Bjerregaard P, Gyntelberg F, Borch- Johnsen K. Prevalence of the metabolic syndrome among the Inuit in Greenland. A comparison between two proposed definitions. Diabet Med 2004;21:1237-42.

10. Brasil. Ministério da Saúde. Política Nacional de Promoção da Saúde 2006. Braślia: Ministério da Saúde; 2006.

11. World Health Organization (WHO). Fifty-seven World Health Assembly. Global Strategy on Diet, Physical Activity and Health. Geneva: WHO, 2004

12. Carvalho T, Nóbrega ACL, Lazzoli JK, Magni JRT, Resende L, et al. Posição oficial da Sociedade Brasileira de Medicina do Esporte: atividade física e saúde. Rev Bras Med Esporte 1996;2:79-81.

13. Matsudo SM, Matsudo VR, Araújo T, Andrade D, Andrade E, Oliveira L, et al. Nível de atividade física da população do estado de São Paulo: análise de acordo com o sexo, idade, nível sócio-econômico, distribuição geográfica e de conhecimento. Rev Bras Cienc Mov 2002;10:41-50.

14. Almeida PM, Wickerhauser H. O critério ABA-ABIPEME: em busca de uma atualização. São Paulo: Associação Brasileira de Anunciantes/Associação Brasileira dos Institutos de Pesquisa de Mercado (ABIPEME), 1991.

15. World Health Organization. Obesity: preventing and managing the global epidemic. Report of a WHO consultation on obesity. Geneva: WHO, 1998.

16. Sociedade Brasileira de Hipertensão (SBH). V Diretrizes de Hipertensão arterial. São Paulo: SBH, 2006

17. Oliveira EP, de Souza MLA, de Lima MDA. Prevalência de síndrome metabólica em uma área rural do semi-árido baiano. Arq Bras Endocrinol Metab 2006;50:456-65.
18. Vargas ER, Vinas MDA, Delisle H. Prevalence of the metabolic syndrome and associated lifestyles in adult males from Oaxaca, Mexico. Salud Publica Mex. 2007;49:94-102.

19. Meigs JB, Wilson PW, Nathan DM, D'Agostino RB, Williams K, Haffner SM. Prevalence and characteristics of the metabolic syndrome in the San Antonio Heart and Framingham Offspring Studies. Diabetes $2003 ;: 2160-7$

20. Ninomiya T, Kubo M, Dói Y, Yonemoto K, Tanizaki Y, Rahman, et al. Impacto f metabolic syndrome on the development of cardiovascular disease in a general Japanese population. The Hisavama Study. Stroke 2007:2063-9.

21. Sociedade Brasileira de Hipertensão. I Diretriz Brasileira de Diagnóstico e Tratamento da Síndrome Metabólica. Hipertensão 2004:130-59.

22. Gupta R, Deedwania PC, Gupta A, Rastogi S, Panwar RB, Kothari K. Prevalence of metabolic syndrome in an Indian urban population. Int J Cardiol 2004;97:257-61.

23. Medina-Lezama J, Zea-Diaz H, Morey-Vargas OL, Bolanos-Salazar JF, Munoz-Atahualpa E, Postigo-Macdowall $\mathrm{M}$, et al. Prevalence of the metabolic syndrome in Peruvian Andean hispanics: The PREVENCION study. Diabetes Res Clin Pract 2007;78:270-81.

24. Saely Ch, Aczel S, Marte T, Langer P, Hoefle G, Drexel H. The metabolic syndrome, insulin resistance, and cardiovascular risk in diabetic and nondiabetic patients. J Clin Endocrinol Metab 2005;90:5698-703.

25. Pitsavos C, Panagiotakos D, Weinem M, Stefanadis C. Diet, exercise and the metabolic syndrome. Rev Diabet Stud 2006;3:118-26.

26. Ciolac GE, Guimarães VG. Exercício físico e síndrome metabólica. Rev Bras Med Esporte 2004;10:319-24.

27. Doro, AR, Gimeno SGA, Hirai AT, Franco LJ, Ferreira SRG. Análise da Associação de Atividade Física à Síndrome Metabólica em Estudo Populacional de Nipo-Brasileiros. Arq Bras Endocrinol Metabol 2006;50:1066-74.

28. Pontes LM, Sousa MSC, Lima RT, Campos RD, Gomes ERM, Santos GL, et al. Prevalência de fatores de risco para doenças crônicas não transmissíveis: impacto de 16 semanas de treinamento futebolístico em índices do estado nutricional e da aptidão física de praticantes de futebol society. Rev Bras Med Esporte 2006;12:211-5.

29. Santos R, Nunes A, Ribeiro JC, Santos P, Duarte JAR, Mota J. Obesidade, síndrome metabólica e atividade física: estudo exploratório realizado com adultos de ambos os sexos, da llha de S. Miguel, Região Autônoma dos Açores, Portugal. Rev Bras Educ Fís Esp 2005;19:317-28.

30. Meigs JB, Wilson PW, Fox CS, Vasan RS, Nathan DM, Sullivan LM, et al. Body mass index, metabolic syndrome, and risk of type 2 diabetes or cardiovascular disease. J Clin Endocrinol Metab 2006;91:2906-12.

31. Geloneze B, Repetto EM, Geloneze SR, Tambascia MA, Ermetice MN. The threshold value for insulin resistance (HOMA-IR) in an admixtured population. IR in the Brazilian Metabolic Syndrome Study. Diabetes Res Clin Pract 2006;72:219-20.

32. Carr MC, Brunzell JD. Abdominal obesity and dyslipidemia in the metabolic syndrome: importance of type 2 diabetes and familial combined hyperlipidemia in coronary artery disease risk. J Clin Endocrinol Metab 2004;89:2601-7. 